\title{
FIRST-PRINCIPLE STUDY OF ELECTRONIC AND VIBRATIONAL PROPERTIES OF LATERAL HETEROSTRUCTURE BASED ON TRANSITION METAL DICHALCOGENIDE (TMDS)
}

\author{
Willian Oliveira Santos ${ }^{1}$, Djardiel da Silva Gomes ${ }^{2}$, Francisco Carlos de \\ Medeiros Filho ${ }^{3}$, Ketly dos Santos Nascimento ${ }^{4}$, José Robson da Costa \\ Venâncio ${ }^{5}$, Nilton Ferreira Frazão ${ }^{6}$ \\ ${ }^{1}$ Mestrando em Física, Universidade Federal de Campina Grande, Campina Grande-PB, Brasil. \\ 2 Doutorando em Física, Universidade Federal da Paraíba, João Pessoa-PB, Brasil. \\ ${ }^{3}$ Mestrando em Ciências Naturais e Biotecnologia, Universidade Federal de Campina Grande, \\ Cuité-PB, Brasil. \\ ${ }^{4}$ Curso de Licenciatura em Física, Unidade Acadêmica Física e Matemática, Universidade \\ Federal de Campina Grande, Cuité-PB, Brasil. \\ ${ }^{5}$ Doutorando em Física, Universidade Federal de Alagoas, Maceió-AL, Brasil. \\ ${ }^{6}$ Prof Unidade Acadêmica de Física e Matemática, Universidade Federal de Campina Grande, \\ Cuité-PB, Brasil. \\ Email para correspondência: willnp 96@hotmail.com
}

\begin{abstract}
Resumo
A compreensão de novos materiais nanoestruturados é muito importante para o avanço tecnológico. Espera-se que a junção de materiais provoque mudanças nas propriedades eletrônicas em comparação com as propriedades desses materiais isolados. Logo, estudar as propriedades físicas após a junção de materiais são importantes para criação de instrumentos cada vez menores e melhores. Neste trabalho, utilizando cálculos de primeiros princípios, implementados com o formalismo da DFT no código computacional CASTEP, investigamos as propriedades eletrônicas das heteroestrutura lateral (HSL) formadas pela junção de planos baseados em dicalcogenetos de metal de transição (TMDs). Como resultado foi visto que HSL apresenta característica semicondutora de gap indireto.
\end{abstract}

Palavras-chave: heteroestrutura lateral, DFT, CASTEP, TMDs.

\begin{abstract}
Understanding new nanostructured materials are very important for technological advancement. The joining of materials is expected to cause changes in electronic properties compared to the properties of these isolated materials. Therefore, studying the physical properties after the joining of materials is important for creating increasingly smaller and better instruments. In this work, using calculations of first principles, implemented with the DFT formalism in the computer code CASTEP, we investigated the electronic properties of the lateral heterostructure $(\mathrm{HSL})$ formed by the joining of plans based on transition metal
\end{abstract}


dichalcogenides (TMDs). As a result, it was seen that HSL has an indirect gap semiconductor characteristic.

Keywords: lateral heterostructure, DFT, CASTEP, TMDs.

\section{Introduction}

As the discovery of graphene by Geim and Novoselov in 2004, through the exfoliation of graphite, a new field of research in two-dimensional (2D) materials in nanostructure was unveiled due to its remarkable properties (NOVOSELOV, et al., 2004, 2005a, 2005b, 2007, 2012; BALANDIN, et al., 2008; LEE, WE, KYSAR,. 2008; KIM, et al., 2011; GEORGAKILAS, et al., 2012). Among this new line of research is the layered transition metal dichalcogenides (TMDs), which are a class of materials with impressive and technologically useful properties, with the MX2 formula, where $M$ represents transition metals and $X$ chalcogen, they can be metals, semiconductors or even superconductors with direct, or indirect band intervals. Several TMD have been extensively investigated experimentally and theoretically for example: MoX2 and WX2 with $(X=S$, Se, and Te) among others (KUC, ZIBOUCHE, HEINE, 2011; KADANTSEV, HAWRYLAK, 2012; BUTLER, et al., 2013; SHI, ZHANG, YAKOBSON, 2013; LEE, et al., 2013; HUANG, DA , LIANG, 2013; LI, et al., 2013; WICKRAMARATNE , ZAHID, LAKE, 2014).

Different TMDs can be combined to form heterostructures (HSs). These are classified as vertical type or lateral type. Vertical HS is by far the most studied type. These are formed by planes of two-dimensional materials stacked via Van der Walls force. However, the number of experimental and theoretical studies with lateral heterostructures (HSL) has been growing strongly due to the emergence and improvement of experimental techniques, such as mechanical exfoliation and chemical vapor decomposition (RUITAO, et al., 2015).

Currently, there are successful experiments with heterojunctions of graphene-hBN, graphene-TMDs, hBN-TMDs, and different combinations of TMD-TMD. Therefore in this work, we seek to build Lateral Heterostructure, in such a way to join materials based on TMD-TMD, with the semiconductorsemiconductor ordering, for that we will make use of the density functional theory, to calculate and analyze the electronic properties of this HSL.

The objective of this work is to build a lateral heterostructure based on RuX2 type TMD ( $X=$ Se and Te), to make an analysis of the HSL electronic and 
thermal properties and compare the results obtained for the isolated monolayers. Which is based on the Density Functional Theory, it is implemented in the computational package CASTEP.

\section{Materials and methods}

The techniques used to study the crystallographic nanostructures of the RuX2 and HSL type TMDs are based on quantum calculations implemented in workstations and supercomputers designed exclusively for scientific computing.

CASTEP is a state-of-the-art program based on quantum mechanics and designed specifically for science of solid-state materials. CASTEP employs the density functional theory and the flat wave method with pseudopotential.

The initial network parameters for RuX2 with ( $X=S e$ and $T e)$ were obtained from the X-ray data provided by Lutz et al. The pyrite structure is composed of 12 atoms in its unit cell, whose spatial group is Pa3 (205) of cubic symmetry. The experimental parameters of the crystal network (angles) RuSe2 are $\mathrm{a}=\mathrm{b}=\mathrm{c}=5,933 \AA$ and for RuTe2 are $\mathrm{a}=\mathrm{b}=\mathrm{c}=6,390 \AA$ ( $\alpha=\beta=\mathrm{y}=90$ ), providing a cell volume equal to $173,74 \AA$ Å.

All calculations were performed within the formalism of Functional Density Theory (DFT) (HOHENBERG, KOHN, 1964; KOHN, SHAM, 1965), performed on the CASTEP plane-wave code (SEGALL, et al., 2002), using the Generalized Gradient Approximation (GGA) (CORSO, et al., 1996; FUCHS, et al., 1998), as an exchange-correlation functional. The functional GGA uses parameterization proposed by Perdew-Burke-Ernzerhof (PERDEW, et al., 1992; PERDEW, BURKE, ERNZERHOF 1996). The integration through the Brillouin zone was carried out employing k-points of sampling using a $5 \times 5 \times 1$, which was sufficient to guarantee the convergence of the electronic structure. The convergence parameters for all geometric optimizations of the cell unit were: total energy variation less than $2.0 \times 10-5 \mathrm{eV}$, maximum force less than 0.05 $\mathrm{eV} / \AA \AA$, maximum voltage component less than $0.1 \mathrm{GPa}$, and maximum displacement less than $2.0 \times 10-2 \AA$.

\section{Results and discussions}

For a better understanding of our results, we divided this section into two subsections. First we will address the electronic properties for RuX2 type 
crystalline structure with $(X=S e$ and $T e)$ separated and its lateral junction; second, we will present the results thermodynamic properties. The results were achieved through ab initio calculations, obtained through the formalism of the density functional theory (DFT), using GGA-PBE approximations. The CASTEP software (Cambridge Sequential Total Energy Package) was used to perform the theoretical calculations.

\subsection{Results of Electronic Properties}

In this section, the results obtained for the electronic structure of some TMDs of the RuX2 type ( $X=S e$ and $T e$ ) will be presented, and later the result obtained for HLS of the RuSe2-RuTe2 type, which presents a semiconductor behavior. It is only worth mentioning that, semiconductors are solids in which they are at an absolute zero temperature, the electrons occupy all the states available in the valence band. In such a way that the conductivity of the semiconductors is caused by the excitation of electrons from the valence band to the conduction band. In addition, the amount of energy required to promote an electron from the valence band to the conduction band is what determines whether a solid will be a conductor, a semiconductor, or an insulator. In Figure 1, we can observe a single cell of the TMDs.
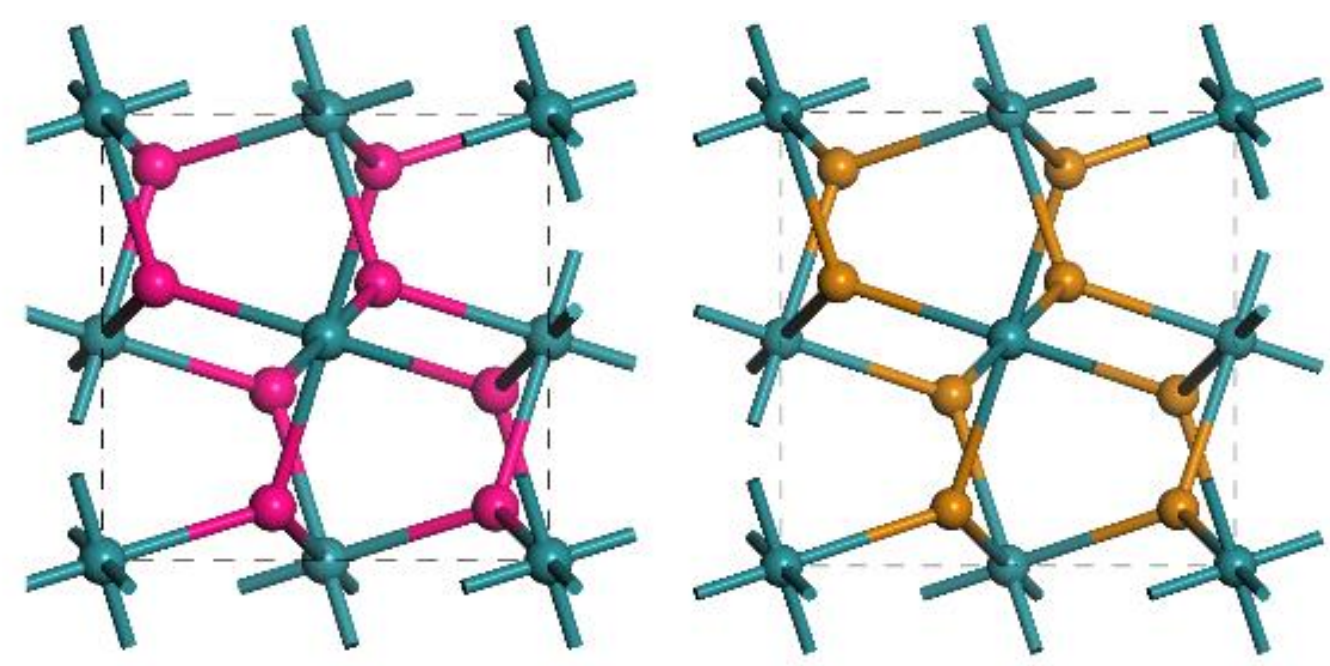

Figure 1: Representation of the unit cell of $\mathrm{RuX}_{2}$ structures ( $\mathrm{X}=\mathrm{Se}$ and Te) Source: Research production 2020

We can see in the graphs shown in figure 2 that both materials have characteristics similar to semiconductor materials. The maximum of the valence 
band occurs at point $\mathrm{M}$ point in the reciprocal space to $\mathrm{RuX}$ and the minimum of the conduction band, for both polyps, is at point $\mathrm{M}$, we can still note that for the RuTe2 type system there is a smaller amount of energy needed for the electron to make this electronic transition compared to the RuSe 2 type system.
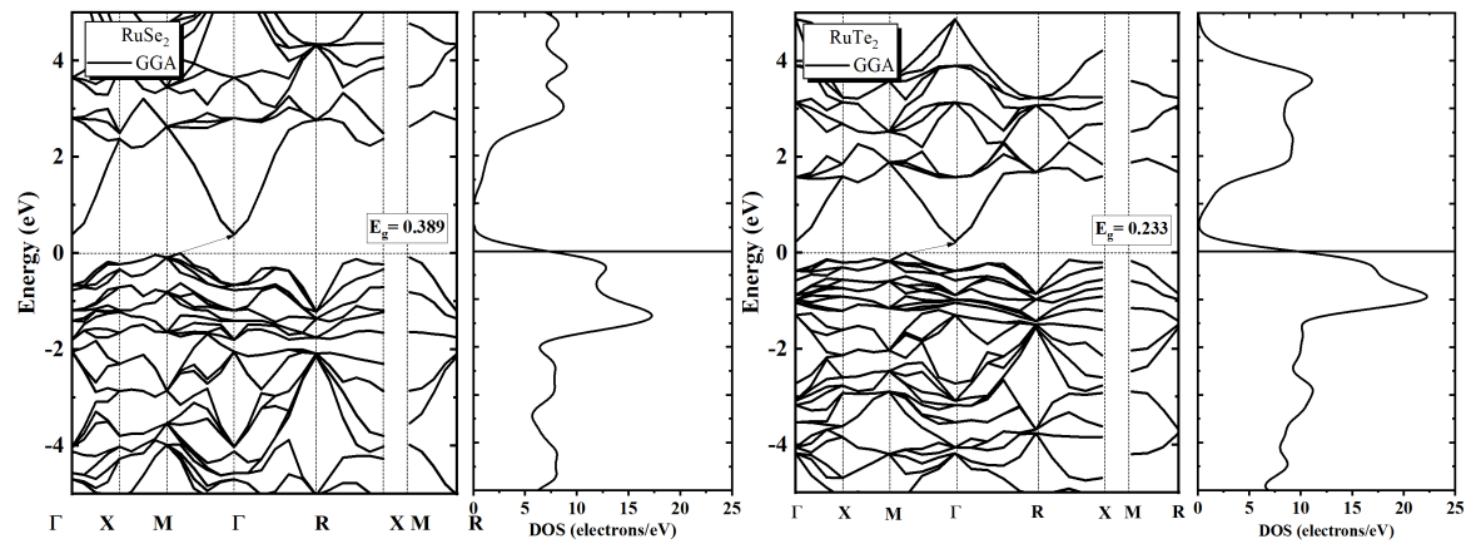

Figure 2: Graphical representation of the energy band structure of the RuX2 monolayers $(X=S e$ and Te). With an emphasis on direct energy gaps for semiconductors

Source: Research production 2020

Analyzing the RuX2 TMDs band structure, we can see that both materials have a narrow and indirect gap semiconductor behavior, as well as, one can see that the energy required for an electronic transition to $\mathrm{RuSe}_{2}$ is 0.389 and for the RuTe2 type system. The value obtained was 0.233 , we can note that in the range -4 to $4 \mathrm{eV}$, for $\mathrm{RuSe}_{2}$ including a set of 12 (12) valence bands from -4 $\mathrm{eV}$ to $0 \mathrm{eV}$ and a set of 6 (12) conduction bands, in the case of the RuTe 2 system we have 13 (12) valence bands from $-5 \mathrm{eV}$ to $0 \mathrm{eV}$ and a set of 6 (12) conduction bands.

Subsequently, we combine the TMD plans to understand the electronic properties of this heterostructure. After defining the interface combination, we use the computer package CASTEP to produce the heterostructures, as shown in figure 3 


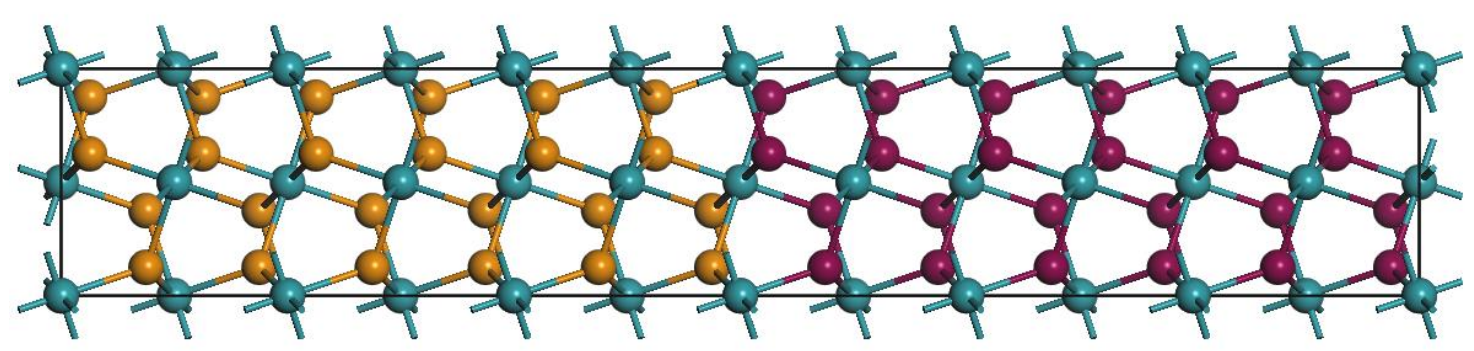

Figure 3: Representation of the unit cell of the Lateral Heterostructure structures for $\operatorname{RuX2}(\mathrm{X}=\mathrm{Se}$ and Te)

Source: Research production 2020

To begin the theoretical study of lateral heterostructures, we chose a path along the first Brillouin Zone to obtain the band structure and Kohn Sham, Z (0.000 0.0000 .500$), \Gamma\left(\begin{array}{lll}0.000 & 0.000 & 0.000\end{array}\right), Y(0.0000 .5000 .000), A(-0.500$ $0.5000 .000), B(-0.5000 .0000 .000), D(-0.5000 .0000 .500), E(-0.5000 .500$ $0.500)$ and $C(0.0000 .5000 .500)$, in this way we calculate the band structure and state density, as can be seen in Figure 4.

Analyzing the results obtained in the band structure of Fig. 4 we notice that our lateral heterojunction has semiconductor characteristics of indirect gap since the maximum of the valence band and the minimum of the conduction band are not aligned. We can also observe that the energy required for the electronic transition to happen is $0.382 \mathrm{eV}$, so we can conclude that our investigated system is a narrow gap semiconductor.

Comparing the results obtained in the isolated monolayers and HLS, we realize that $98 \%$ of the RuSe 2 monolayer gap is similar in the heterojunction in compensation in the case of RuTe2 $61 \%$. Thatis, in the isolated RuSe2 monolayer, we obtain an almost equal value in the lateral junction, but for RuTe2, we had a much higher value compared to its monolayer. So we have that in the electronic structure of the heterojunction has a greater contribution of the isolated monolayer of $\mathrm{RuSe}_{2}$. 


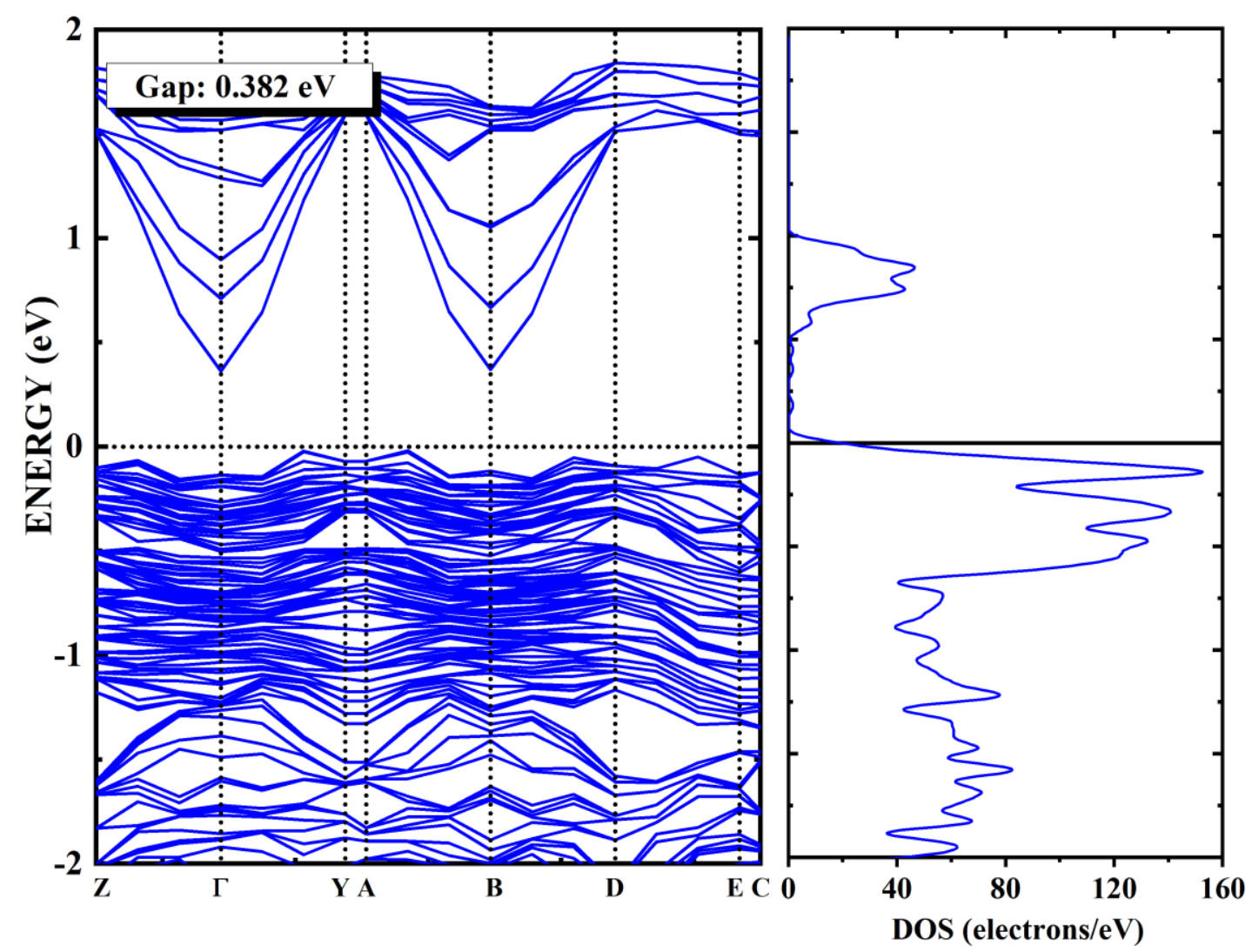

Figure 4: Graphical representation of the energy band structure of the HLS RuSe ${ }_{2}-\mathrm{RuTe}_{2}$. Calculated in the DFT formalism, using the CASTEP program with the functional GGA

Source: Research production 2020

We also note that the valence band has two local maximums and the conduction band is analogous, where it has two local minimums. The explanation for the appearance of this phenomenon is that we are joining two indirect gap semiconductors. We can also see that in the HLS band structure, the electronic transition can occur to both point $\Gamma$ and point $B$.

\subsection{Thermodynamic properties}

The thermal properties of the crystalline system are associated with the vibrational properties of the crystalline network, contains essential information for its possible technological applications.

The descriptions of the properties of the independent systems can be carried out through some thermodynamic potentials. That said, the stability of our systems is also verified by the thermodynamic potentials, where we calculate Enthalpy $(H)$, Free Energy $(G)$, and T x S (Temperature $x$ Entropy), in such a way that $T \times S=U F(U$ is the internal energy and $F$ is Helmholtz's free 
energy). We also obtained the thermal capacity at constant volume $\left(\mathrm{C}_{\mathrm{v}}\right)$ and the temperature of Debye $(\theta D)$.

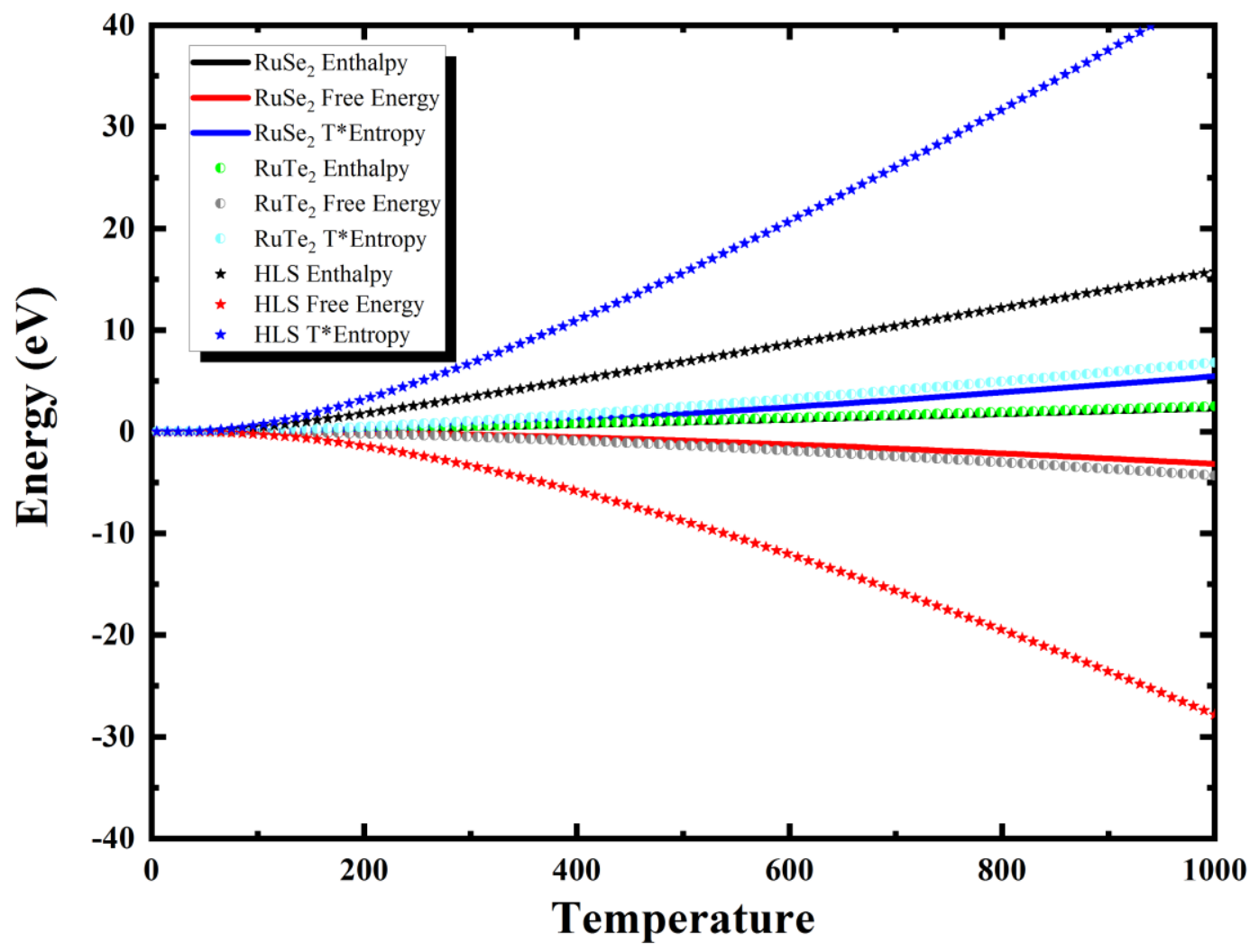

Figure 5: Enthalpy, Free Energy and Temperature $x$ Entropy of RuX2 and HSL

Source: Research production 2020

In Figure 5, we show the enthalpy profiles of the calculated thermodynamic potentials, free energy, and temperature times the entropy term, as a function of temperature (in $\mathrm{K}$ ), $\mathrm{RuX} 2$ for $\mathrm{HLS}$, for comparison purposes. It can be seen that the enthalpy (blue line) has an almost linear behavior for RuSe2. Now for RuTe2, we can notice assuming higher energy values as a function of temperature, and with regard to HLS, it possible to see that the enthalpy value (blue star) has higher values than isolated systems.

The free energy for RuSe2 (red line) and RuTe2 (gray ball) both behaviors are decreasing rapidly, up to $200 \mathrm{~K}$, as the temperature increases for both structures, so for HLS the behavior is analogous, but after $200 \mathrm{~K}$ they get value higher compared to the result of the separate system. The term TxS for both $\mathrm{RuX}_{2}$ and HSL plots increases more exponentially as a function of temperature for both crystalline structures. In addition, analyzing an entropy and enthalpy for 
each structure, we can conclude that the synthesis process for both phases is non-spontaneous.

The temperature of Debye is a parameter that serves to ensure the rigidity of the material, that is, material without flexibility, so the higher the Debye temperature of the material, the more strongly coupled the atoms of the crystalline network will meet. Where consequently, we will have a decreased thermal vibration between the atoms involved. In the figure below, we have the thermal capacity and the temperature of Debye of the investigated systems.
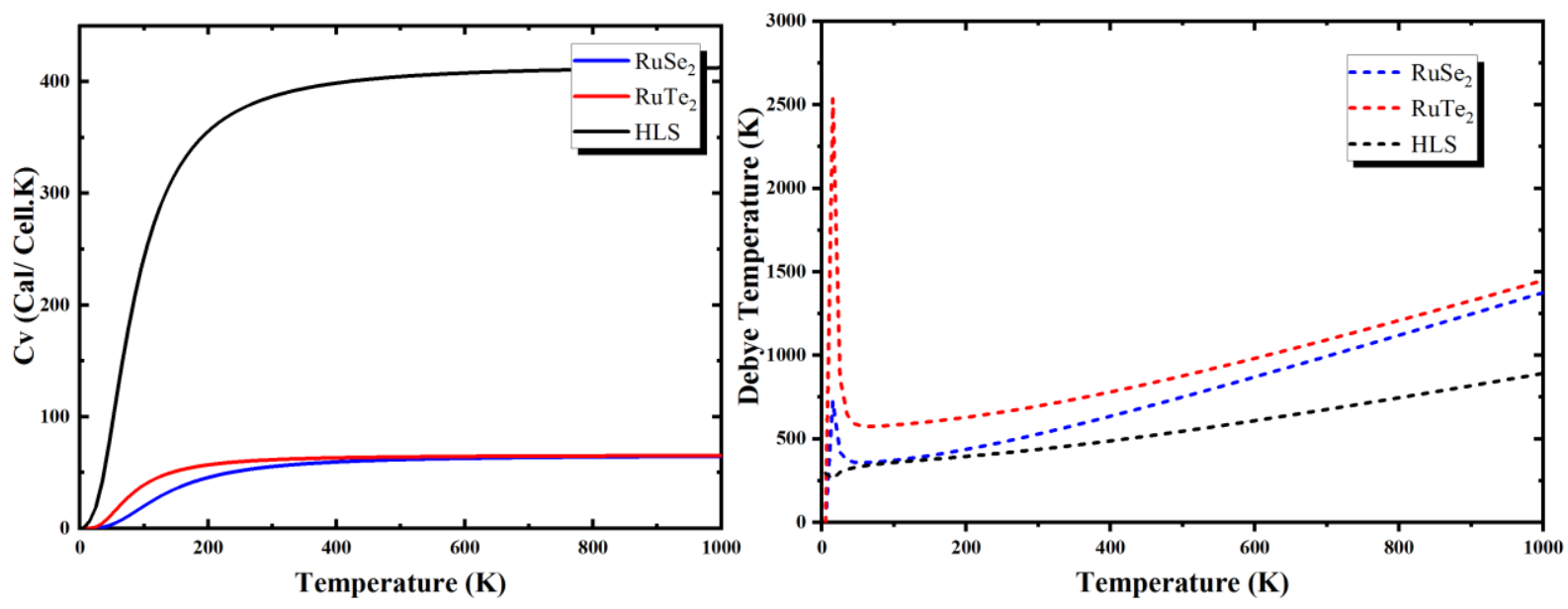

Figure 6: Thermal capacity at constant volume (left scale) and temperature Debye (right scale) as a function of the temperature $(K)$ of the systems Source: Research production 2020

Observing the picture on the left where it exposes $\theta D$, one could observe that the crystalline structure of the RuSe2 type has atoms more strongly linked than $\mathrm{RuTe}_{2}$ because the value obtained for a higher Debye temperature. Comparing the separate $\theta_{D}$ values with that of the HLS, we can conclude that the $\mathrm{RuSe}_{2}$ structure will have less is the thermal vibration than $\mathrm{RuTe}_{2}$ and HLS.

The thermal capacity of a body is defined as a quantity that quantifies the ratio between the amounts of heat received by the body and the temperature variation. For for both $\mathrm{RuX}_{2}$ systems, we have similar behavior for $\mathrm{C}_{\mathrm{v}}$. regarding the lateral junction of the materials, we figure out a huge increase in value for $\mathrm{C}_{\mathrm{v}}$.

\section{Conclusion}

We will present the main results and conclusions obtained in this work, through quantum calculations based on Density Functional Theory (DFT), using the 
GGA-PBE approximation for calculating electronic and vibrational properties, $\mathrm{RuX} \mathrm{X}_{2} \operatorname{com}(\mathrm{X}=\mathrm{Se}$ e Te$)$ and side junction (HLS). For this, we use the CASTEP module, which is based on the density functional theory, and to minimize the calculation time, it uses the method of the total potential of flat waves, and Pseudopotentials to represent the system.

We realized that when we analyzed the results obtained, through the theory of the density functional with the use of the functional GGA-PBE, for the electronic properties of the monolayers isolated from transition metal dichhalide dicalcogenides and for the lateral heterostructure (HSL) formed by the heterojunction of the two-dimensional planes of TMDC based materials with $\mathrm{RuX} \mathrm{X}_{2}$ semiconductor characteristics ( $\mathrm{X}=\mathrm{Se}$ and $\left.\mathrm{Te}\right)$.

In the electronic properties of structure (BS) of bands and state density (DOS) for the monolayers we see that the materials that present semiconductor characteristics, where for $\mathrm{RuSe}_{2}$ we find the indirect gaps of $0.389 \mathrm{eV}$ and for $\mathrm{RuTe}_{2}$ crystalline system $0.233 \mathrm{eV}$ and HLS $0.382 \mathrm{eV}$. Regarding the mechanical stability of our systems, we calculate the thermodynamic properties, we observe that the thermal capacity and temperature of Debye increase as the temperature increases, which confirms the rigidity of the studied systems.

\section{References}

Balandin A. A, et al .Superior thermal conductivity of single-layer graphene, Nano Lett. , 8 (2008), p. 902. Access in: April 15, 2020.

Butler S. Z., et al. Progress, challenges and opportunities in two-dimensional materials in addition to graphene, ACS Nano , 7 (2013), p. 2898. Access in: August 9, 2020.

Corso D., et al. Generalized-gradient approximations to density-functional theory: A comparative study for atoms and solids. Physical Review B, v. 53, n. 3, p. 1180, 1996.

Fuchs M., et al. Pseudopotential study of binding properties of solids within generalized gradient approximations: The role of core-valence exchange correlation. Physical Review B, v. 57, n. 4, p. 2134, 1998. 
Georgakilas V., et al .Functionalization of graphene: covalent and non-covalent approaches, derivatives and applications, Chem. Rev., 112 (2012), p. 6156. Access in: April 15, 2020.

Hohenberg P., Kohn W. Teoria do funcional da densidade (DFT). Phys. Rev , v. 136, p. B864, 1964. Access in: August 9, 2020.

Huang W., Da H., Liang G. Electronic, transport and optical properties of bulk and single-layer PdSe 2, J. Appl. Phys. , 113 (2013), p. 104304. Access in: August 9, 2020.

Kadantsev E. S., Hawrylak P. Electronic structure of a single monolayer MoS 2, Solid State Commun. , 152 (2012), p. 909. Access in: August 9, 2020.

Kim K., et al. A role of graphene in silicon-based semiconductor devices, Nature, 479 (2011), p. 338. Access in: April 15, 2020.

Kohn W., Sham L. J. Self-consistent equations including exchange and correlation effects. Physical review, v. 140, n. 4A, p. A1133, 1965.

Kuc A., Zibouche N., Heine T. Influence of quantum confinement on the electronic structure of TS 2 transition metal sulfide, Phys. Rev. B, 83 (2011), p. 245213. Access in: August 9, 2020.

Lee C. G; Wei X. D; Kysar J. W. Hone Measurement of the elastic properties and intrinsic strength of monolayer graphene, Science, 321 (2008), p. 385. Access in: April 15, 2020.

Lee C., et al. Improving the thermoelectric properties of transition metal dichhalides in $2 \mathrm{H}-\mathrm{MQ} 2$ layers $(\mathrm{M}=\mathrm{Mo}, \mathrm{W} ; \mathrm{Q}=\mathrm{S}, \mathrm{Se}, \mathrm{Te})$ by layer mixing: functional investigation of density, Chem. Mater. , 25 (2013), p. 3745. Access in: August 9, 2020.

Li W., et al.Density functional theory and beyond for band-gap screening: performance for transition metal oxides and dichalcogenides J. Chem. Comput Theory. , 9 (2013), p. 2950. Access in: August 9, 2020. 
Lutz H.; et al. Refinement of the structure of the pyridadisulfide type of ruthenium, rus2, and ruthenium diselenide, ruse2.Sta Acta CrystallographicaC: Communications of the crystalline structure, International Union of Crystallography, v. 46, n. 11, p.20032005, 1990. Access in: August 9, 2020.

Novoselov K. S, et al. Two-dimensional gas from massless dirac fermions in graphene, Nature, 438 ( 2005a ) , p. 197. Access in: April 15, 2020.

Novoselov K. S, et al. A script for graphene, Nature, 490 (2012), p. 192. Access in: April 15, 2020. Access in: August 9, 2020.

Novoselov K. S., et al. Electric field effect in thin atomic carbon films, Science, 306 ( 2004 ) , p. 666. Access in: April 15, 2020.

Novoselov K. S., et al. Quantum effect of ambient temperature on graphene, Science, 315 ( 2007 ) , p. 1379. Access in: April 15, 2020.

Novoselov K. S., et al. Two-dimensional atomic crystals, Proc. Natl. Acad. Sci. EUA, 102 ( 2005b) , p. 10451.

Perdew J. P. et al. Atoms, molecules, solids, and surfaces: Applications of the generalized gradient approximation for exchange and correlation. Physical review B, v. 46 , n. 11, p. $6671,1992$.

Perdew J. P.; Burke K.; Ernzerhof M. Generalized gradient approximation made simple. Physical review letters, v. 77, n. 18, p. 3865, 1996.

Ruitao L. V., et al. Transition Metal Dichalcogenides and Beyond: Synthesis, Properties, and Applications of Single- and Few-Layer Nanosheets. Acc. Chem. Res., 48 (1): 56-64, Jan 2015. Access in: August 9, 2020.

Segall, M. D. et al. First-principles simulation: ideas, illustrations and the CASTEP code. Journal of physics: condensed matter, v. 14, n. 11, p. 2717, 2002.

Shi H., Pan H., Zhang Y. W., Yakobson B. I. Quasiparticle band structures and optical properties of tensioned monolayer MoS 2 and WS 2, Phys. Rev. B, 87 (2013), p. 155304. Access in: August 9, 2020. 
Wickramaratne D., Zahid F., Lake R. K. Electronic and thermoelectric properties of lowlayer transition metal dichhalides, J. Chem. Phys. , 140 (2014), p. 124710. Access in: August 9, 2020.

\section{Acknowledgments}

To the Laboratory of Computational Simulation and Modeling of Nanomaterials (LABMOL), located in the Center for Education and Health of the Federal University of Campina Grande (UFCG- CES), Cuité campus, for the space ceded that allowed the processing of calculations that culminated in the results of this work. To the UFCG Graduate Program in Physics (PPG Physics) and the Coordination for the Improvement of Higher Education Personnel Brazil (CAPES) for the granting of the scholarship. 